\title{
NOUVELLE
}

\section{La polypose juvénile infantile serait causée par une délétion de la région 10q23 comprenant les deux gènes suppresseurs de tumeurs BMPRIA et PTEN}

Capucine Delnatte, Damien Sanlaville, Jean-François Mougenot, Dominique Stoppa-Lyonnet
C. Delnatte, D. Stoppa-Lyonnet: Département de Génétique, Institut Curie, 26, rue d'Ulm, 75248 Paris Cedex 5, France.

D. Sanlaville: Département de Génétique,

Hôpital Necker-Enfants Malades, AP-HP, Paris, France et Hospices Civils de Lyon, Service de Cytogénétique constitutionnelle, Hôpital Edouard Herriot, Lyon, France. J.F. Mougenot : Département de Pédiatrie, Hôpital NeckerEnfants Malades, AP-HP, Paris, France.

dominique.stoppa-lyonnet@curie.net
> La polypose juvénile se caractérise par la présence de multiples polypes juvéniles dans le tractus gastro-intestinal, prédisposant non seulement à la survenue de cancers colo-rectaux mais aussi, dans une moindre mesure, de tumeurs gastriques [1]. Elle a été subdivisée en trois groupes par Sachatello en 1975 [2] : la polypose juvénile colique, la polypose juvénile généralisée et la polypose juvénile infantile [2]. Ces trois types se différencient essentiellement par l'âge au diagnostic, la localisation des polypes et la sévérité de la symptomatologie digestive. La polypose juvénile infantile définie par Sachatello et al. est caractérisée par la survenue des symptômes avant l'âge de 6 ans, l'atteinte généralisée du tractus digestif et par la gravité de la symptomatologie pouvant mettre en jeu le pronostic vital [2]. Les deux premiers types de polypose juvénile sont associés, dans environ $50 \%$ des cas, à des mutations constitutionnelles hétérozygotes des gènes BMPRIA ou SMAD4 [3]. Aucun gène n'a été identifié jusque-là dans les cas de polypose juvénile infantile. L'absence de forme familiale a con- duit Sachatello et al. à évoquer une transmission autosomique récessive.

Nous avons caractérisé sur le plan génétique quatre enfants atteints de polypose juvénile infantile [4]. S'ajoutaient aux tableaux digestifs, dans trois cas, une macrocéphalie, une légère dysmorphie faciale et - pour l'un des enfants - la présence de lipomes et d'hémangiomes. L'association de ces signes cliniques nous avait fait évoquer dans un premier temps le diagnostic de syndrome de Bannayan-Riley-Ruvalcaba. Afin de confirmer cette hypothèse diagnostique, nous

\section{Point de cassure centromérique}

Point de cassure télomérique

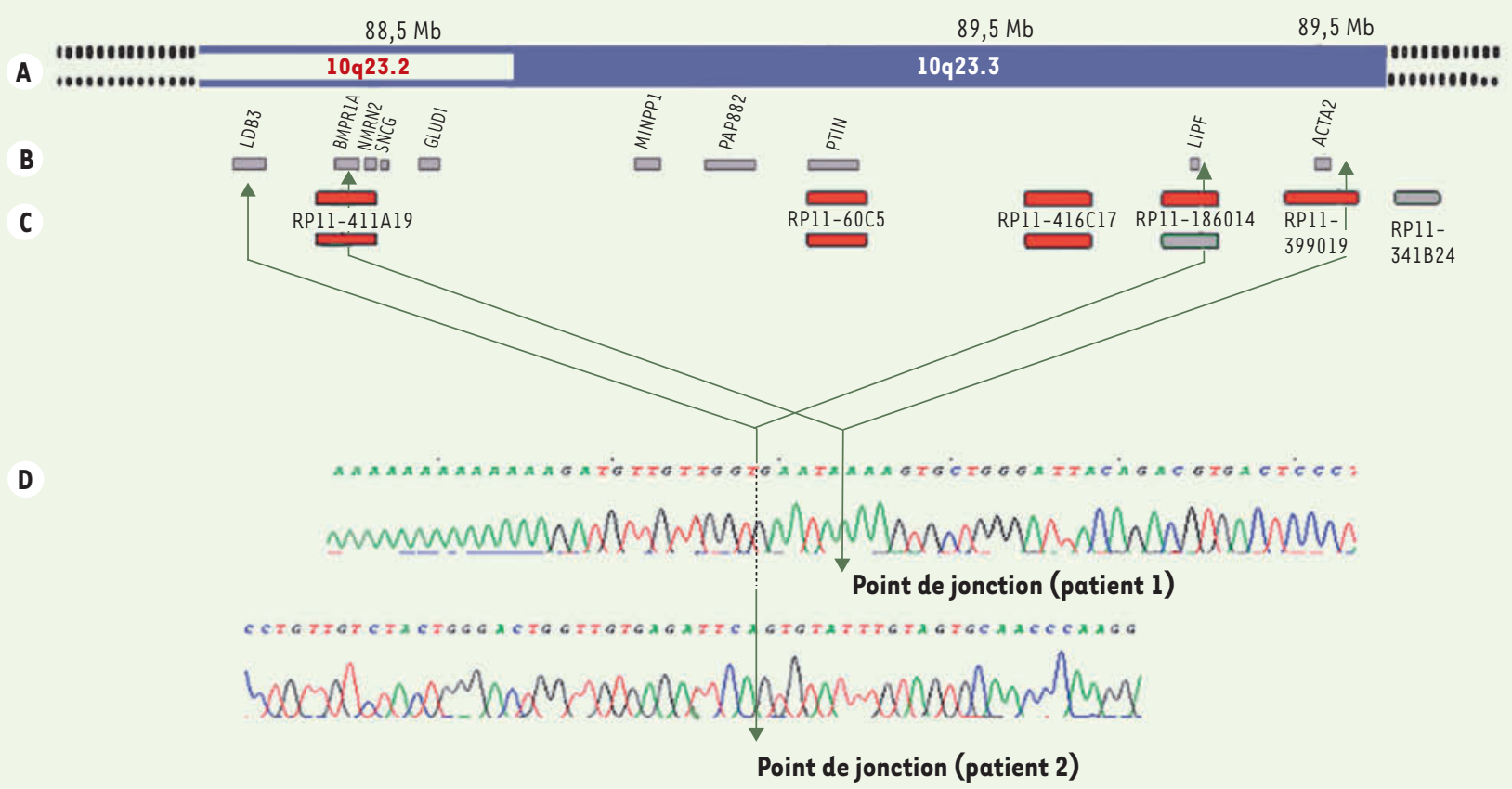

Figure 1. Localisation des points de cassures mis en évidence chez les patients 1 et 2. A. Carte physique de la région 10q23 (depuis Ensembl version 35). B. Gènes localisés dans la région délétée. C. Clones utilisés pour l'étude cytogénétique (en rouge : délétés; en vert : présents). D. Séquences indiquant le point de jonction. 
avons recherché dans ces trois cas une mutation ponctuelle du gène $P T E N$, gène impliqué dans ce syndrome. Cette recherche s'est révélée négative. Mais une étude familiale indirecte, réalisée dans deux cas pour lesquels nous disposions de l'ADN des parents, a montré la non-contribution de marqueurs de l'un des deux parents, situés dans la région chromosomique du gène PTEN, suggérant une délétion de novo du gène (d'origine paternelle pour l'un des enfants et maternelle pour l'autre). Cette délétion du gène PTEN a été confirmée par hybridation in situ en fluorescence (FISH). Nous avons alors recherché, par cette même technique, si la délétion concernait aussi le gène BMPRIA. En effet, ce gène impliqué dans les polyposes juvéniles coliques et généralisées est situé à environ $1 \mathrm{Mb}$ du gène PTEN. Les résultats obtenus par FISH ont mis en évidence la délétion du gène BMPRIA chez ces deux enfants. La taille et les bornes de ces deux délétions ont été finement caractérisées. La recherche s'est effectuée en deux temps. Dans un premier temps, les techniques de CGH-array et de FISH nous ont permis de localiser les bornes centromériques des délétions dans une région de $200 \mathrm{~kb}$ et les bornes télomériques dans une région de $300 \mathrm{~kb}$. Dans un second temps, nous avons utilisé une technique de PCR semi-quantitative, récemment décrite, la PCR multiplexe/chromatographie liquide (MP/ LC) [5] afin de préciser, au nucléotide près, les bornes des deux délétions. Cette technique utilise l'ADN-chromatographie liquide haute performance (DHPLC). Elle permet de quantifier le nombre de copies de régions génomiques, I'ADN des deux parents ayant été utilisé comme témoin. Cette technique a été menée pas à pas dans les régions précédemment définies par les techniques de FISH et CGH-array. Pour chaque cas, nous avons pu restreindre à quelques $\mathrm{kb}$ la localisation des bornes de la délétion, ce qui nous a permis de faire une PCR long range et, après séquençage du produit de $P C R$, de caractériser au nucléotide près l'étendue de chaque délétion. II s'agit dans chaque cas d'une délétion de $2 \mathrm{Mb}$ n'impliquant pas les mêmes points de cassure (Figure 1). Chaque délétion emporte un certain nombre de gènes et de transcrits dont PTEN et BMPRAl restent les deux candidats pour rendre compte du phénotype clinique présenté par ces jeunes patients.

Les deux autres cas correspondent également à une délétion constitutionnelle de ces deux gènes. Les bornes des délétions n'ont cependant pas été définies au nucléotide près. Dans un cas, il s'agit d'une mosaïque visible au moins dans les cellules du sang périphérique. II est possible qu'au niveau digestif, compte tenu de la gravité du tableau, il n'y ait pas de mosaïque. Nous proposons que la polypose juvénile infantile, telle que l'a définie Sachatello, soit secondaire à la délétion de deux gènes contigus: $P T E N$ et BMPRIA. Des données de la littérature confortent cette hypothèse. En effet, quatre enfants présentant un tableau de polypose juvénile infantile sont porteurs d'une délétion sur le chromosome 10q dont la définition cytogénétique est compatible avec une délétion de ces deux gènes (pour revue, voir [4]).

Nous avons fait l'hypothèse selon laquelle la sévérité du phénotype digestif de la polypose juvénile infantile est secondaire à la synergie entre les voies de signalisation auquel appartiennent les deux gènes, respectivement la voie TGF- $\beta$ /BMP pour BMPRIA et PI3K/AKT pour PTEN. En effet, il existe des arguments biologiques en faveur de l'influence de la voie TGF- $\beta$ /BMP sur la voie PI3K/AKT. L'exposition de lignées de cancer du sein MCF-7 à BMP2 provoque l'augmentation du taux de protéine PTEN consécutive à une diminution de sa dégradation relayée par le protéasome [6]. De plus, il a été montré que la voie BMP, dans les cellules souches intestinales, régulait négativement la $\beta$-caténine, cela passant par la régulation positive de l'activité de PTEN [7]. Or, la $\beta$-caténine a un rôle dans la prolifération cellulaire [8]. II est donc probable qu'une double délétion des gènes BMPRIA et PTEN entraîne une diminution de l'activité de la voie TGF- $\beta$ /BMP, voie connue pour inhiber la prolifération cellulaire, et une augmentation de l'activité de la voie PI3K/AKT, voie impliquée dans la survie, la croissance et la prolifération cellulaire. Ainsi, il est vraisemblable qu'il existe une véritable coopération entre ces deux voies, majorant l'effet de la perte de chacune et conduisant alors à un tableau clinique digestif sévère. Pour confirmer de façon définitive que le substratum génétique de la polypose juvénile infantile est la double délétion des gènes $B M P R I A$ et PTEN, il reste à caractériser au niveau moléculaire les cas d'autres enfants atteints. II est à noter qu'à la suite de notre publication, une équipe italienne a rapporté le cas d'un enfant ayant une délétion de $12 \mathrm{Mb}$ comprenant les gènes $P T E N$ et $B M P R I A$ et qui présente une polypose juvénile infantile, mais dont le phénotype est paradoxalement, compte tenu de la taille de la délétion, plus atténué que ceux que nous avons rapportés [9]. Dans notre réponse, nous avons fait l'hypothèse que la taille de la délétion est telle que des gènes modificateurs interviennent pour permettre la viabilité cellulaire et celle de l'individu, conduisant ainsi à un phénotype atténué, ces gènes pouvant être non liés aux locus BMPRIA et PTEN, ou pouvant être en trans de la région délétée, sur l'allèle restant [10]. $\diamond$ Contiguous gene deletion within chromosome arm 10q is associated with juvenile polyposis of infancy, reflecting cooperation between the BMPRIA and PTEN tumor-suppressor genes

\section{REMERCIEMENTS}

Nous remercions l'ensemble des auteurs qui ont contribué à notre étude : Joris-Robert Vermeesch, Claude Houdayer, Marie-Christine de Blois, David Genevieve, Olivier Goulet, Jean-Pierre Fryns, Francis Jaubert, Michel Vekemans, Stanislas Lyonnet, Serge Romana, Charis Eng.

\section{RÉFÉRENCES}

1. Howe JR, Mitros FA, Summers RW. The risk of gastrointestinal carcinoma in familial juvenile polyposis. Ann Surg Oncol 1998 ; 5 : 751-6.

2. Sachatello CR, Griffen WO Jr. Hereditary polypoid diseases of the gastrointestinal tract: a working classification. Am J Surg 1975 ; 129 : 198-203.

3. Howe JR, Sayed MG, Ahmed AF, et al. The prevalence of MADH4 and BMPRIA mutations in juvenile polyposis and absence of BMPR2, BMPR1B, and ACVR1 mutations. J Med Genet $2004 ; 41: 484-91$.

4. Delnatte C, Sanlaville D, Mougenot JF, et al. Contiguous gene deletion within chromosome arm 10q is associated with juvenile polyposis of infancy, reflecting cooperation between the BMPRIA and PTEN tumor-suppressor genes. Am J Hum Genet 2006 ; 78 : 1066-74.

5. Dehainault C, Lauge A, Caux-Moncoutier V, et al. Multiplex $\mathrm{PCR} /$ liquid chromatography assay for detection of gene rearrangements: application to $\mathrm{RBl}$ gene. Nucleic Acids Res $2004 ; 32$ : el39.

6. Waite KA, Eng C. BMP2 exposure results in decreased PTEN protein degradation and increased PTEN levels. Hum Mol Genet $2003 ; 12: 679-84$.

7. He XC, Zhang J, Tong WG, et al. BMP signaling inhibits intestinal stem cell self-renewal through suppression of Wnt-beta-catenin signaling. Nat Genet 2004 ; 36 : 1117-21.

8. Gregorieff A, Clevers H. Wnt signaling in the intestinal epithelium: from endoderm to cancer. Genes Dev 2005 ; $19: 877-90$.

9. Salviati L, Patricelli M, Guariso G, et al. Deletion of PTEN and BMPRIA on chromosome $10 \mathrm{q} 23$ is not always associated with juvenile polyposis of infancy. AmJ Hum Genet 2006 ; 79 : 593-6.

10. Sanlaville D, Delnatte C, Mougenot JF, et al. Reply to Salviati et al. Am J Hum Genet 2006 ; 79 : 596-7. 